\title{
Prevalence and determinants of type 2 diabetes mellitus in a Greek adult population
}

\author{
Sofia Tsirona, Fotis Katsaros, Alexandra Bargiota, Stergios A. Polyzos, \\ George Arapoglou, George N. Koukoulis
}

Department of Endocrinology and Metabolic Diseases, University Hospital, School of Medicine, University of Thessaly, Biopolis, Larissa, Greece

\begin{abstract}
The prevalence of diabetes mellitus (DM) is increasing worldwide reaching epidemic proportions. The aim of the present study was to estimate the prevalence of DM in Thessaly, a large region of Central Greece, and to extrapolate our results to the population of the entire country. A random sample of 805 adults (421 females and 384 men) living in Thessaly, aged 18-80 years, was surveyed. After completing a questionnaire about health status and a thorough physical examination, a blood sample was obtained from each participant for biochemical analysis. Participants with fasting glucose levels between 100-125 mg/dl underwent an oral glucose tolerance test (OGTT). A second survey was also conducted, via telephone call-interviews, in a randomly selected sample age- and sex-stratified to the country's adult population in order to extrapolate the DM data from Thessaly to the whole country. The frequency of DM based on patient history and fasting blood glucose levels was $6.96 \%$, comparable to that observed in the telephone-based nationwide survey $(7.38 \%, p=0.669)$. However, after the OGTT an additional $3.72 \%$ of the population had undiagnosed DM, increasing DM prevalence to $10.68 \%$ (age adjusted $11.77 \%$ ). The prevalence of pre-diabetes was $8.70 \%$, with impaired fasting glucose at $5.84 \%$ and impaired glucose tolerance at $2.86 \%$. The prevalence of DM was significantly higher in men $(14.58 \%)$ than in women $(7.13 \%, p<0.001)$, increased with age in both sexes and was more prevalent in hypertensive $(p<0.001)$ and obese subjects $(p=0.001)$ and in those living in rural areas $(p=0.003)$. In the multiple logistic regression analysis, significant predictors of pre-diabetes and DM together were age, homeostasis model of assessment of insulin resistance (HOMA-IR), alcohol consumption and educational status, whereas those of DM alone were age, HOMA-IR and triglycerides. Extrapolating our data to the whole country, the age-adjusted prevalence of DM was estimated at $11.97 \%$ (men $13.98 \%$, women $9.25 \%$ ), clearly indicating a major public health problem.
\end{abstract}

Key words: Diabetes mellitus, Frequency, Greece, OGTT, Prevalence, Thessaly 


\section{INTRODUCTION}

Diabetes mellitus (DM) is a metabolic disorder characterized by a constellation of abnormalities of glucose homeostasis, which are associated with significant acute and chronic complications. It constitutes a global health problem, as the number of people with $\mathrm{DM}$ is increasing worldwide, this due to population growth, aging, urbanization, lifestyle modifications and the increasing prevalence of obesity and physical inactivity. ${ }^{1}$ Currently, DM has reached epidemic proportions; the prevalence of DM worldwide was $2.8 \%$ in the year $2000,6.4 \%$ in 2010 and is predicted to reach $7.7 \%$ by $2030,{ }^{2-4}$ while the prevalence of impaired glucose tolerance (IGT) was $7.9 \%$ in 2010 and is predicted to be $8.4 \%$ by $2030 .{ }^{2-4}$ IGT is a serious condition, considering that pre-diabetic individuals may develop type $2 \mathrm{DM}$ in the subsequent 10 years and are also at higher risk for cardiovascular diseases than normoglycemic individuals. ${ }^{5,6}$

Data from different studies indicate that the prevalence of DM varies widely worldwide among populations due to differences in genetic susceptibility and environmental factors. ${ }^{7}$ In Greece, only a limited number of population-based studies have been conducted on the prevalence of DM. Most of these studies concern small areas, either rural or urban, are based on self-reported data and are retrospective or pertain to non-randomly selected populations.

The aim of this study was to estimate the prevalence of DM and IGT in the population of Thessaly, a large region of Central Greece. Secondary aims of the study were: a) to determine factors related to DM and b) to extrapolate the results to the entire Greek population using the self-reported data from a nationwide survey.

\section{SUBJECTS AND METHODS}

The study was carried out in accordance with the Declaration of Helsinki. Written informed consent was signed by all the participants and the study protocol was reviewed and approved by the scientific and ethics committee of the University Hospital of Larissa.

\section{Sample selection}

The sample of the study consisted of 805 individuals, 421 females and 384 males, who were drawn from the Adult Regional Greek Obesity Survey (ARGOS), ${ }^{8}$ a community-based health and nutrition study. This survey was carried out in the region of Thessaly, Central Greece, with a population of approximately 625,000 adult inhabitants, aged $18-80$ years, living in urban $(60 \%)$ or rural $(40 \%)$ areas for at least one year. The details of the random sample selection were described in our previous study. ${ }^{8}$ Briefly, the sample was randomly selected from the capital city of each of the four provinces of Thessaly, as well as from other towns and villages, in proportion to their population. The baseline evaluation included: demographic characteristics, age, gender, profession, personal and family history of DM, hypertension, hypercholesterolemia, smoking, dietary and alcohol consumption habits. According to the population census, areas with less than 2,000 inhabitants were defined as rural. ${ }^{9}$ The data were collected from 2003 to 2005.

In order to extrapolate these data to the prevalence of DM throughout the country, we conducted a second survey in a randomly selected sample, age- and sex-stratified to the country's adult population according to the census of 2001 of the National Service for Statistics. ${ }^{9}$ The communication was performed via telephone call-interviews and was selected by a List-Assisted Random Digit Dialing (RDD) sampling method. Interviews were performed with 10,000 individuals (aged 18-80 years). The sample size for the whole country's population survey was estimated according to power analysis accepting a confidence level of $99 \%$, a margin of error of $1.5 \%$ and a response distribution of $50 \%$. From the 10,000 adults reached by telephone, 7,238 agreed to participate in the study (refusal rate 28\%).

Each subject was asked whether he/she had any problem with their blood glucose level. In the subgroup of individuals who responded that they had $\mathrm{DM}$, a second telephone-based interview was conducted which included questions about demographic and socioeconomic characteristics, duration of DM, physical activity, kind of treatment, etc. This study was conducted from 24/9/2008 to 2/10/2008.

\section{Procedure}

The participants from the region of Thessaly were asked to come to the nearest public health center in the morning (8.30-9.30 am) after having fasted for 
at least 12 hours. Subjects were asked to bring all of their medications.

Each subject underwent a thorough physical examination. Body weight and height, waist and hip circumference and blood pressure were measured as previously described. ${ }^{8}$ A blood sample was obtained from all the participants for the measurement of glucose, insulin, total cholesterol, high density lipoprotein cholesterol (HDL-C) and triglycerides.

The blood samples were centrifuged on site and the serum and plasma specimens were transferred immediately, in cold boxes filled with ice, to the university hospital laboratories and stored at $-70^{\circ} \mathrm{C}$ until the measurement. In addition, each participant was asked by the doctor about his/her health status and health behaviors (smoking, alcohol intake, dietary habits, etc.).

Subjects with fasting plasma glucose (FPG) levels $\geq 126 \mathrm{mg} / \mathrm{dl}$ in two consequent measurements were considered as diabetics and those with FPG levels $<100 \mathrm{mg} /$ dl normoglycemic. Participants with FPG levels between 100 and $125 \mathrm{mg} / \mathrm{dl}$ were considered to have impaired fasting glucose (IFG) and 64 out of 100 subjects with IFG underwent an oral glucose tolerance test (OGTT) after 3 days of ad libitum carbohydrate diet. Subjects were classified as being diabetics, with IGT or IFG, when the 2-hour plasma glucose level was $\geq 200 \mathrm{mg} / \mathrm{dL}, \geq 140$ but $<200 \mathrm{mg} / \mathrm{dL}$ or $<140 \mathrm{mg} / \mathrm{dl}$, respectively, according to the WHO criteria. ${ }^{10}$ Pre-diabetes is a term used to describe a high-risk state for the development of diabetes. ${ }^{11}$ It is assumed that pre-diabetes is the combination of IFG and IGT. For the quantification of insulin resistance, the homeostasis model of assessment of insulin resistance (HOMA-IR) was calculated. ${ }^{12}$ Fasting total cholesterol levels $\geq 200 \mathrm{mg} / \mathrm{dl}$, triglycerides levels $\geq 150 \mathrm{mg} / \mathrm{dl}$ and HDL-C levels $<40 \mathrm{mg} / \mathrm{dl}$ for men and $<50 \mathrm{mg} / \mathrm{dl}$ for women were respectively defined as hypercholesterolemia, hypertriglyceridemia and low HDL cholesterol according to the National Cholesterol Educational Program Adult Treatment Panel III guidelines..$^{13}$ Participants using lipid lowering agents were considered as having hyperlipidemia.

Body mass index (BMI; $\left.\mathrm{kg} / \mathrm{m}^{2}\right)$ was calculated from the body weight and height according to Quetelet's formula: $\mathrm{BMI}=$ weight $(\mathrm{kg}) /$ height $^{2}\left(\mathrm{~m}^{2}\right) .{ }^{14}$ Waist circumference (WC) and the calculated waist-to-hip ratio (WHR) were used as estimates of central obesity. Abdominal obesity was defined by a WC greater than $102 \mathrm{~cm}$ for men and greater than $88 \mathrm{~cm}$ for women. ${ }^{15}$ Weight status was classified according to the WHO definitions: ${ }^{16}$ underweight (UW): $\mathrm{BMI}<18.5$, normal weight (NW): BMI 18.5 - 24.9, overweight (OW): BMI 25 - 29.9, obese: BMI $\geq 30$ and extremely or morbidly obese (MO): $\mathrm{BMI} \geq 40$. Hypertension was defined as blood pressure $\geq 140$ or $\geq 90 \mathrm{mmHg}$ for systolic or diastolic blood pressure, respectively, or the use of antihypertensive medication. Those who had smoked for over a year and those who had stopped smoking during the last year were defined as smokers. The rest were non-smokers.

\section{Laboratory analysis}

Plasma insulin levels were measured using a commercially available immunoradiometric assay (IRMA) kit (Immunotech Beckman Counter Company, Marseille, France, PW). The assay's sensitivity for insulin measurements was $0.51 \mathrm{IU} / \mathrm{mL}$ and the interassay coefficient of variation (CV) $3.4 \%$ without any crossreactivity with proinsulin. Total cholesterol, HDL-C, triglycerides and glucose were measured by standard methods on an automatic analyzer (Olympus 600; Medicon, Athens, Greece). Specifically regarding plasma glucose, this was measured by an automated enzymatic assay (hexokinase). LDL-C was calculated according to the Friedwald equation.

\section{STATISTICAL ANALYSIS}

Results are presented as mean \pm standard deviation (SD) for normally distributed continuous variables, as median (range) for non-normally distributed continuous variables and as frequencies for qualitative variables. The Kolmogorov-Smirnov test was used to check the normality of distributions of the continuous variables. The independent sample Student's t-test or the MannWhitney U-test was used to compare between group differences for continuous variables when there were two groups. The chi-square test or Fisher's exact test was used to compare between group differences for qualitative variables. The Cochran-Armitage test was also used to explore whether there was a clear trend of DM rate with age and BMI, treated as categorical variables. One-way ANOVA, with Bonferonni cor- 
rection for multiple pairewise comparisons, was used to compare between group differences for continuous variables when there were more than two groups. The age-adjusted prevalence of DM, IFG, IGT and prediabetes was calculated by the direct method. The mid-year (2007 and 2008) population estimates by sex and age groups was used as a "standard" population. ${ }^{17}$

Multiple logistic regression analysis ("stepwise" method) was performed to identify variables independently associated with DM. All variables that provided $\mathrm{p}$-value $<0.01$ in univariate analysis were entered in each model of multiple regression analysis. Variables that were not normally distributed were logarithmically transformed for the need of these analyses. A two-sided $p$-value $<0.05$ was considered statistically significant in all the above tests. Regression analyses were performed with SPSS 21.0 for Macintosh (IBM Corp., Armonk, NY, USA).

\section{RESULTS}

A total of 805 individuals (384 (47.7\%) men and 421 (52.3\%) women), aged 47.0 \pm 13.3 , were included in the study. According to BMI, 29.3\% had normal weight, $40.5 \%$ were overweight and $30.2 \%$ were obese (men: $17.7 \%, 51.1 \%$, and $31.2 \%$; women: $39.9 \%, 30.9 \%$, and $29.2 \%$, respectively). Abdominal obesity was present in $42.5 \%$ (39.1\% of men and $45.6 \%$ of women).

The prevalence of DM, based on patient history and fasting plasma glucose levels, was $6.96 \%$, without any significant difference between males and females (Table 1). $12.42 \%$ of individuals had IFG, with significantly higher rates in men compared to women (Table 1). In individuals with IFG who underwent an OGTT, we found that $29.70 \%$ of them were diabetics, $23.40 \%$ had IGT and the remaining $46.90 \%$ had IFG (Table 2). Similar rates of undiagnosed DM were observed in men and women, despite a non-significant trend towards higher rates in men. After extrapolating the frequency of DM, IGT and IFG to the whole number of subjects with fasting plasma glucose $100-125 \mathrm{mg} /$ $\mathrm{dl}$, it was found that an additional $3.72 \%$ of subjects had undiagnosed DM, increasing the overall prevalence of DM in our sample population to $10.68 \%$ with significantly higher frequency in men compared to women $(14.58 \%$ vs. $7.13 \%, p<0.001)$ (Table 3$)$. The overall prevalence of pre-diabetes was $8.70 \%$ (IFG $5.84 \%$ and IGT $2.86 \%$ ), with significantly higher frequency of IFG and pre-diabetes in males compared to females (Table 3).

In the group of 7,238 subjects interviewed by telephone, known DM was declared by 531 (7.38\%),

Table 1. Prevalence of normoglycemia (NG), impaired fasting glucose (IFG) and diabetes mellitus (DM) based on fasting plasma glucose levels

\begin{tabular}{|c|c|c|c|c|c|c|}
\hline \multirow[b]{2}{*}{ Plasma glucose levels (mg/dl) } & \multicolumn{2}{|c|}{ Males } & \multicolumn{2}{|c|}{ Females } & \multicolumn{2}{|c|}{ Total } \\
\hline & $\mathbf{n}$ & $\%$ & $\mathbf{n}$ & $\%$ & $\mathbf{n}$ & $\%$ \\
\hline$<100(\mathrm{NG})$ & $280 / 384$ & 72.92 & $369 / 421$ & $87.65^{*}$ & $649 / 805$ & 80.62 \\
\hline 100-125 (IFG) & $72 / 384$ & 18.75 & $28 / 421$ & $6.65^{*}$ & $100 / 805$ & 12.42 \\
\hline$\geq 126(\mathrm{DM})$ & $32 / 384$ & 8.33 & $24 / 421$ & 5.70 & $56 / 805$ & 6.96 \\
\hline
\end{tabular}

$*_{\mathrm{p}}<0.001$ compared to males

Table 2. The frequency distribution of IFG, IGT and DM in the 64 subjects who underwent OGTT

\begin{tabular}{|c|c|c|c|c|c|c|}
\hline \multirow[b]{2}{*}{ Plasma glucose levels (mg/dl) } & \multicolumn{2}{|c|}{ Males } & \multicolumn{2}{|c|}{ Females } & \multicolumn{2}{|c|}{ Total } \\
\hline & n & $\%$ & n & $\%$ & $\mathrm{n}$ & $\%$ \\
\hline 2 hours after the 75 gr glucose load & 46 & & 18 & & 64 & \\
\hline$<140(\mathrm{IFG})$ & 21 & 45.65 & 9 & 50.00 & 30 & 46.90 \\
\hline 140-199 (IGT) & 10 & 21.74 & 5 & 27.78 & 15 & 23.40 \\
\hline$\geq 200(\mathrm{DM})$ & 15 & 32.61 & 4 & 22.22 & 19 & 29.70 \\
\hline
\end{tabular}


Table 3. Prevalence of NG, IFG, IGT and DM in our group after adjusting for the frequency in the total number of subjects with impaired fasting glucose

\begin{tabular}{|c|c|c|c|c|c|c|}
\hline & \multicolumn{2}{|c|}{ Males } & \multicolumn{2}{|c|}{ Females } & \multicolumn{2}{|c|}{ Total } \\
\hline & n & $\%$ & n & $\%$ & $\mathrm{n}$ & $\%$ \\
\hline $\mathrm{NG}$ & 280 & 72.92 & 369 & $87.65^{*}$ & 649 & 80.62 \\
\hline IFG & 33 & 8.59 & 14 & $3.32 * *$ & 47 & 5.84 \\
\hline IGT & 15 & 3.91 & 8 & 1.90 & 23 & 2.86 \\
\hline DM & 56 & 14.58 & 30 & $7.13^{*}$ & 86 & 10.68 \\
\hline
\end{tabular}

${ }^{*} \mathrm{p}<0.001,{ }^{* *} \mathrm{p}=0.002$ compared to males

indicating a prevalence comparable to that found in Thessaly $(p=0.669)$. After adjustment for age, the prevalence of DM in Thessaly was found to be $11.77 \%$, whereas after extrapolating these data to the whole country, the age-standardized prevalence of DM was calculated at $11.97 \%$ (Table 4 ).

The subsequent analyses included 769 subjects after exclusion of those who declined to undergo the OGTT. The prevalence of DM was found to increase significantly with age $(\mathrm{p}=0.001)$ in both sexes, reaching its highest level in the group of 70-80 year-olds without any significant difference between men and women (Figure 1, Table 5). Rural residents had a significantly higher prevalence of DM than urban residents; when it was examined separately, women living in rural regions had significantly higher rates of DM, whereas statistical significance was marginally lost among men. However, this difference was no longer significant after controlling for age. The prevalence

Table 4. The age-adjusted prevalence of diabetes mellitus (DM), impaired glucose tolerance (IGT), impaired fasting glucose (IFG) and pre-diabetes in adults aged 20-79 in Thessaly and the whole country

$\begin{array}{lccc}\text { DM } & \text { IGT } & \text { IFG } & \text { Pre-diabetes } \\ (\%) & (\%) & (\%) & (\%)\end{array}$

\begin{tabular}{lcccc}
\hline Thessaly & & & & \\
Male & 13.70 & 4.17 & 9.62 & 13.79 \\
Female & 9.20 & 2.13 & 2.64 & 4.77 \\
Total & 11.77 & 3.01 & 5.90 & 8.91 \\
Greece & & & & \\
Male & 13.98 & 4.01 & 9.57 & 13.58 \\
Female & 9.25 & 1.90 & 2.76 & 4.66 \\
Total & 11.97 & 2.81 & 6.03 & 8.84 \\
\hline
\end{tabular}

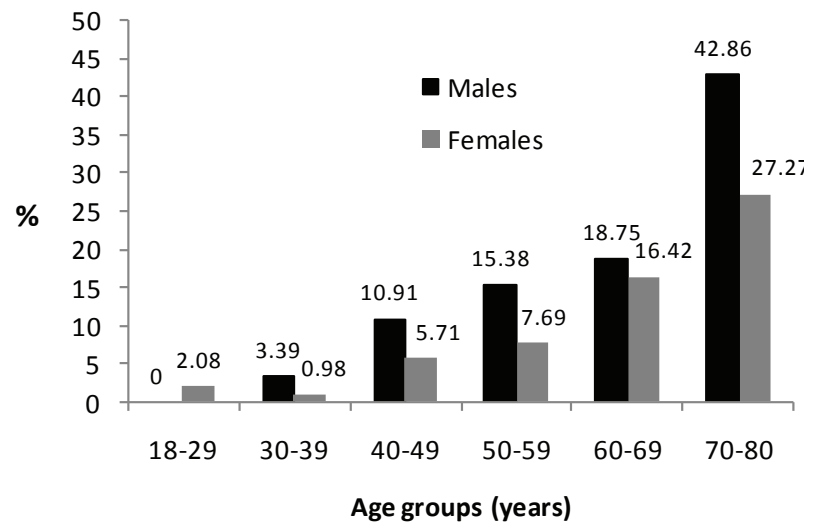

Figure 1. The rate of diabetes in males and females in the various age groups.

of DM was significantly higher in married than in single individuals, but it lost statistical significance when tested separately in men and women (Table 5). Education inversely influenced the frequency of DM: participants with a lower educational level (primary education) had a significantly higher prevalence of DM (Table 5) compared to those with a higher educational level (secondary and tertiary).

Comparative data regarding cardiovascular risk factors are presented in Table 6. As expected, men and women with higher BMI or abdominal obesity had a significantly higher prevalence of DM. On the other hand, subjects with DM or IGT had significantly higher BMI and WC compared to individuals with normoglycemia (31.51 \pm 6.64 vs. $27.72 \pm 5.24$ $\mathrm{kg} / \mathrm{m}^{2}, \mathrm{p}=0.001 ; 106.57 \pm 13.15$ vs $93.37 \pm 14.79 \mathrm{~cm}$, $\mathrm{p}=0.001$ and $30.91 \pm 5.75 \mathrm{vs} 27.72 \pm 5.24 \mathrm{~kg} / \mathrm{m}^{2}, \mathrm{p}=0.006$; $101.03 \pm 12.57$ vs $93.37 \pm 14.79 \mathrm{~cm}, \mathrm{p}=0.003$, respectively). An association was also found between DM and hypertension. Men and women with hypertension had a significantly higher prevalence of DM compared to individuals without hypertension (Table 6). Smoking was also negatively associated with DM rates. Smokers had lower rates of DM compared to non-smokers. Hypercholesterolemia was not associated with DM prevalence, nor were hypertriglyceridemia and low HDL (data not shown).

In multiple logistic regression analysis, age, HOMA-IR, alcohol consumption and educational status were independently associated with the presence of T2DM/pre-diabetes (IFG and IGT) when compared 
Table 5. The prevalence of diabetes mellitus among men and women based on sociodemographic characteristics

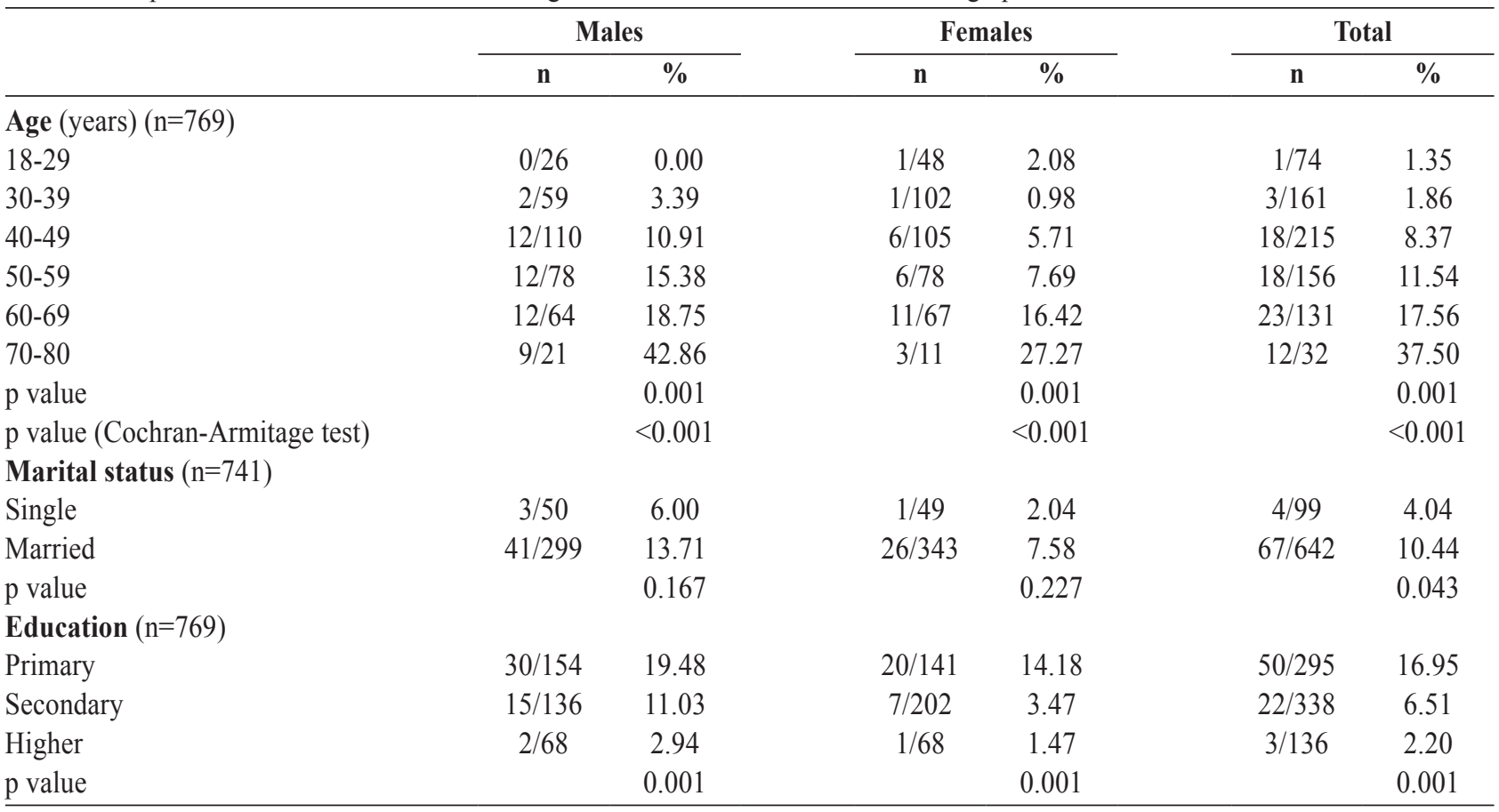

Table 6. Prevalence of diabetes mellitus among men and women based on cardiovascular risk factors

\begin{tabular}{|c|c|c|c|c|c|c|}
\hline & \multicolumn{2}{|c|}{ Male } & \multicolumn{2}{|c|}{ Female } & \multicolumn{2}{|c|}{ Total } \\
\hline & $\mathbf{n}$ & $\%$ & $\mathbf{n}$ & $\%$ & $\mathbf{n}$ & $\%$ \\
\hline \multicolumn{7}{|l|}{ BMI $\left(\mathrm{kg} / \mathrm{m}^{2}\right)(\mathrm{n}=769)$} \\
\hline $\mathrm{BMI}<25$ & $3 / 62$ & 4.84 & $6 / 162$ & 3.7 & $9 / 224$ & 4 \\
\hline BMI 25-29,9 & $21 / 184$ & 11.41 & $5 / 129$ & 3.88 & $26 / 313$ & 8.3 \\
\hline $\mathrm{BMI} \geq 30$ & $23 / 112$ & 20.53 & $17 / 120$ & 14.17 & $41 / 232$ & 17.67 \\
\hline $\mathrm{p}$ value & & 0.001 & & 0.001 & & 0.001 \\
\hline $\mathrm{p}$ value (Cochran-Armitage test) & & $<0.001$ & & $<0.001$ & & $<0.001$ \\
\hline \multicolumn{7}{|l|}{ Abdominal obesity ( $\mathrm{n}=769$ ) } \\
\hline Yes & $29 / 154$ & 18.83 & 23/199 & 11.56 & $52 / 353$ & 14.73 \\
\hline No & $18 / 204$ & 8.82 & $5 / 212$ & 2.36 & $23 / 416$ & 5.53 \\
\hline $\mathrm{p}$ value & & 0.007 & & $<0.001$ & & $<0.001$ \\
\hline \multicolumn{7}{|l|}{ Hypertension $(\mathrm{mmHg})(\mathrm{n}=769)$} \\
\hline Yes & $28 / 121$ & 23.14 & $12 / 84$ & 14.28 & $39 / 205$ & 19.02 \\
\hline No & $19 / 237$ & 8 & $16 / 327$ & 4.89 & $35 / 564$ & 6.2 \\
\hline $\mathrm{p}$ value & & 0.001 & & 0.006 & & $<0.001$ \\
\hline \multicolumn{7}{|l|}{ Smoking $(n=769)$} \\
\hline Yes & $17 / 180$ & 9.44 & $2 / 102$ & 1.96 & $19 / 282$ & 6.74 \\
\hline No & $30 / 178$ & 16.85 & 26/309 & 8.41 & $56 / 487$ & 11.5 \\
\hline $\mathrm{p}$ value & & 0.042 & & 0.023 & & 0.032 \\
\hline \multicolumn{7}{|l|}{ Hypercholesterolemia $(\mathrm{n}=769)$} \\
\hline Yes & $35 / 254$ & 13.78 & $18 / 263$ & 6.84 & $53 / 517$ & 10.25 \\
\hline No & $12 / 104$ & 11.54 & $10 / 148$ & 6.76 & $22 / 252$ & 8.73 \\
\hline $\mathrm{p}$ value & & 0.610 & & $>0.999$ & & 0.605 \\
\hline
\end{tabular}


with non-diabetic individuals (Table 7); individuals of higher age, HOMA-IR and alcohol consumption, but lower educational status were at higher risk for T2DM. These results remained essentially unchanged when wine consumption replaced alcohol consumption in this regression model.

Age, HOMA-IR and serum triglycerides were independently associated with the presence of T2DM when pre-diabetes (IFG and IGT) was excluded from the analysis (Table 8); individuals of higher age, HOMA-IR and triglycerides were at higher risk for T2DM.

\section{DISCUSSION}

The present study assessed the prevalence of DM in a random sample of adults living in a large region of Central Greece with both urban and rural areas. We found that a large proportion of adults $(10.68 \%$; men $14.58 \%$ and women $7.13 \%$ ) had DM, while a smaller proportion (2.86\%) had IGT and $8.70 \%$ had pre-diabetes. It should be emphasized that DM was undiagnosed in $34.88 \%$ of cases.

Epidemiological data regarding the prevalence of DM are rare in Greece. The first epidemiological studies concerning its prevalence, using glycosuria as a screening method, were conducted in the early 70 's showing low rates of DM. ${ }^{18,19}$ Twenty years later, similar estimates $(1.52 \%)$ were found by Lionis et al in a retrospective study based on reviewing all medical records of a small rural district health care center and performing OGTT in subjects with nondiagnostic values of fasting glucose, based on the WHO criteria. ${ }^{20}$ Our estimated prevalence of DM is in accordance with that of a study synchronous to our own conducted in the urban area of Attica ${ }^{21}$ (DM prevalence $6.74 \%$ ), in subjects free of apparent cardiovascular diseases, indicating a potential selection bias. Compared to another synchronous study conducted in a rural area, including individuals aged 1-99 years, ${ }^{22}$ our DM prevalence is higher $(10.68 \%$ vs. $7.40 \%)$. However, when we restricted their age-range to 20-80 years, the results concerning DM prevalence were comparable to our data in the rural area $(9.35 \%$ vs. $11.52 \%)$. Close to our results is also the prevalence of DM in Cyprus $(10.3 \% ; 6.5 \%$ with known DM and 3.8\% with undiagnosed DM) with population characteristics comparable to those of Greece. ${ }^{23}$

The prevalence of DM seems to be on the increase worldwide, although different racial, cultural, financial and habitual factors may influence its frequency across

Table 7. Multiple logistic regression analysis (stepwise method) for independent associates of diabetes and pre-diabetes vs. healthy controls

\begin{tabular}{lcccc}
\hline Covariate & B coefficient & Adjusted Odds Ratio & p-value & 95\% CI for adjusted odds ratio \\
\hline Age (years) & 0.054 & 1.056 & 0.001 & $1.022-1.090$ \\
HOMA-IR & 0.293 & 1.340 & $<0.001$ & $1.216-1.477$ \\
Alcohol consumption (Y/N) & 0.980 & 2.664 & 0.002 & $1.415-5.017$ \\
Educational status (Lower vs Higher) & -0.591 & 0.554 & 0.029 & $0.326-0.942$
\end{tabular}

Individuals without T2DM or IFG or IGT were rated as 0 and those with T2DM or IFG or IGT as 1 within the dependent variable. Variables initially selected but finally excluded from the equation were: gender, fat mass, BMI, waist circumference, systolic arterial pressure, diastolic arterial pressure, total cholesterol, triglycerides and smoking.

Table 8. Multiple logistic regression analysis (stepwise method) for independent associates of diabetes vs. healthy controls

\begin{tabular}{|c|c|c|c|c|}
\hline Covariate & B coefficient & Adjusted odds ratio & p-value & $95 \%$ CI for adjusted odds ratio \\
\hline Age (years) & 0.082 & 1.086 & $<0.001$ & $1.044-1.129$ \\
\hline HOMA-IR & 0.258 & 1.294 & $<0.001$ & $1.161-1.442$ \\
\hline Triglycerides (mg/dL) & 0.004 & 1.004 & 0.020 & $1.001-1.007$ \\
\hline
\end{tabular}

Individuals without T2DM or IFG or IGT were rated as 0 and those with T2DM as 1 within the dependent variable.

Variables initially selected but finally excluded from the equation were: gender, permanent residence, educational status, smoking status, fat mass, BMI, waist circumference, mean arterial pressure, total cholesterol, HDL-cholesterol. 
the world. In Europe, the known comparative agestandardized prevalence of DM in 2010 ranged from $2.1 \%$ to $12 \%$, according to the International Diabetes Federation. ${ }^{2}$ Using only FPG for the diagnosis, the overall prevalence of DM in Greece (6.96\%) appears to be lower than that documented recently in the USA in adults over 20 years old $(9.3 \%) .{ }^{24}$ Undiagnosed DM has been reported to carry a similar risk of mortality to that of diagnosed DM. ${ }^{25-27}$ However, the estimated prevalence depends on the method used. The WHO 1999 protocol is considered the gold standard for estimating undiagnosed DM, using measurements of FPG and 2-hour glucose. ${ }^{28}$ According to the 2013 IDF Diabetes Atlas, globally, undiagnosed DM is estimated to account for $45.8 \%$ of all cases, with considerable variability across different world regions ranging from $24.5 \%$ to $75.1 \% .{ }^{27}$ This variability is attributed to a number of determinants, among which income is an important one affecting health care and the educational level of citizens. Health systems are generally more developed in high-income countries and thus DM is likely to be detected earlier. However, even within each country, access to health care may be lower in rural compared to urban areas. The proportion of undiagnosed DM ranges in Europe from 30\%, based on FPG ${ }^{29}$ to $44-61 \%$, based on OGTT, in two regional ${ }^{30,31}$ and four nationwide studies. ${ }^{26,32-34}$ Compared to data found in the well-controlled European studies concerning the prevalence of undiagnosed DM based on OGTT, our results are lower (approximately $35 \%$ of DM), possibly indicating more timely diagnosis of DM in Thessaly. Of course, regional differences in the proportion of undiagnosed DM are likely to affect the total prevalence of DM and may partly explain its variability among different countries with a comparable socioeconomic environment. ${ }^{35}$

The OGTT-based studies provide estimates of pre-diabetes (combined IFG and IGT), which is an important risk factor for DM. Regarding the prevalence of pre-diabetes in Greece, to the best of our knowledge, there is only one previously mentioned survey in a rural region, conducted in $2002 .{ }^{22}$ In the agecomparable group of the above study, the prevalence of pre-diabetes was $4.22 \%$, this being similar to our results when we compared the group of participants living in rural areas $(5.56 \%)$. Our results regarding the prevalence of pre-diabetes $(8.7 \%)$ are comparable to those found in Cyprus (9.5\%), but lower than those in the abovementioned European countries where prevalence ranges from $14 \%$ to $30 \%,{ }^{35}$ and the results of the NHANES (2005-2008) survey which showed that the pre-diabetes rate among non-Hispanic whites in the USA was $29.5 \%{ }^{36}$

The prevalence of DM in our cohort was found to be significantly higher in men compared to women, the latter being analogous to published data from Spain. ${ }^{37}$ It was observed that the prevalence of diabetes was higher in rural areas, but after controlling for age, the statistical significance of this difference was lost, confirming previously reported results from Greece. ${ }^{38}$

Age, education, HOMA-R and alcohol consumption were independently associated with $\mathrm{DM} /$ pre-diabetes. When pre-diabetes was excluded from the regression analysis, only age, HOMA-R and triglycerides remained independently associated with DM. Given that this was an observational study, a causal relationship cannot be established; however, we could speculate that alcohol consumption may influence factors that contribute to progression of metabolic disturbances (e.g., inflammation, oxidative stress and/or beta-cell dysfunction). Our results are in accordance with those found in a recent meta-analysis ${ }^{39}$ showing that alcohol consumption of more than $50 \mathrm{gr} /$ day increases the risk for DM. Other risk factors such as obesity and sedentary life associated with alcohol consumption may also explain our results.

Age is an independent risk factor for DM as observed in our series, this concurring with published data from developed countries. ${ }^{40}$ Therefore, the expected increase in life expectancy represents a factor contributing to the worldwide increase of DM.

Lower educational status was also found to be associated with DM in our study. This inverse association is in agreement with published data from developed countries. ${ }^{41,42}$ It should also be stressed that a lower educational level is usually associated with low income.

As expected, HOMA-IR, an index of insulin resistance, was independently associated with DM. Obesity is a major negative determinant of insulin sensitivity, as has been shown in human and animal studies, since weight gain correlates positively with insulin resist- 
ance ${ }^{43}$ Insulin resistance is a key component of DM, driving beta-cells to a compensatory enhancement of insulin secretion until the time they can no longer produce sufficient insulin, resulting initially in IGT and eventually in type $2 \mathrm{DM}^{44}$

In agreement with published data, we found that cardiovascular risk factors, such as obesity, abdominal obesity and hypertension, are more common in diabetic than non-diabetic individuals. ${ }^{45,46}$ Obesity is an established risk factor for DM. ${ }^{47}$ This study showed that the frequency of DM in obese subjects was four times higher compared to individuals with normal weight. This association was also evident among individuals from rural areas, where obesity is significantly higher compared to urban areas. ${ }^{8}$

DM prevalence was lower in smokers than nonsmokers. Other studies have also shown that BMI in smokers is lower than in non-smokers, ${ }^{48}$ indicating that smokers tend to be thinner, this possibly explaining the inverse association of smoking with DM. Furthermore, since this was an observational study, lower rates of DM in smokers may simply indicate that some ex-smokers had stopped smoking after the diagnosis of DM. On the other hand, smoking may play a role in the development of DM, as this is indicated in a recent study showing that smoking cessation in type 2 diabetics was associated with amelioration of their metabolic parameters. ${ }^{49}$ Moreover, smoking is often associated with other unhealthy behaviors such as lack of physical activity, unhealthy diet and high alcohol consumption, factors that favor weight gain ${ }^{50}$ and consequently increase the risk of DM.

Our findings are particularly alarming taking into account that DM is associated with increased incidence of long-term microvascular and macrovascular complications that will inevitably result in higher mortality, as this is indicated in the INTERHEART case-control study in Europians. ${ }^{51}$

The present study has certain limitations. First, this was an observational study, therefore no causeeffect relationship could be established. Second, the region of Thessaly may not accurately represent the whole country, although the prevalence of diagnosed DM in our telephone-conducted survey was not different; thus the extrapolation of our data to the whole country should be accepted with some reservation.
Moreover, the extrapolation of data of 64 subjects subjected to OGTT to the 100 subjects with IFG may have slightly influenced our results. Finally, we did not measure $\mathrm{HbAlc}$ due to limited resources; this might have underestimated the prevalence of DM.

In conclusion, the prevalence of DM and prediabetes in Thessaly seems to be comparable to that observed in other European countries. This should be seriously considered by the Greek Health Agency, since DM-associated complications lead to increased cost, morbidity and mortality. Screening programs may be of importance so as to earlier diagnose individuals who are unaware of being diabetics and thereby more efficiently follow up those with pre-diabetes.

\section{CONFLICT OF INTEREST STATEMENT}

The authors declare that there is no conflict of interest and that they received no specific funding for this article.

\section{REFERENCES}

1. Wild S, Roglic G, Green A, Sicree R, King H, 2004 Global prevalence of diabetes: estimates for the year 2000 and projections for 2030. Diabetes Care 27: 1047-1053.

2. IDF Diabetes Atlas, 4th ed. International Diabetes Federation 2009.

3. IDF Diabetes Atlas, 3th ed. International Diabetes Federation 2007.

4. Shaw JE, Sicree RA, Zimmet PZ, 2010 Global estimates of the prevalence of diabetes for 2010 and 2030. Diabetes Res Clin Pract 87: 4-14.

5. Matfin G, Pratley RE, 2010 Advances in the treatment of prediabetes. Ther Adv Endocrinol Metab 1: 5-14.

6. Buysschaert M, Bergman M, 2011 Definition of prediabetes. Med Clin North Am 95: 289-297.

7. Amot A, McCarty D, Zimmet P, 1997 The rising burden of diabetes and its complications: estimates and projections to the year 2010. Diabetic Medicine 14: Suppl 5: 1-85.

8. Koukoulis GN, Sakka C, Katsaros F, et al, 2010 High rates of obesity prevalence in adults living in central Greece: data from the ARGOS study. Hormones (Athens) 9: 253-262.

9. Population census 2001. General Secretariat of the National Statistical Service of Greece. http://statistics. gr/portal/page/portal/ESYE.

10. Anon Definition, diagnosis and classification of diabetes mellitus and its complications, report of a WHO Consultation. Part 1: Diagnosis and classification of diabetes mellitus. Geneva: WHO, 1999; 1-65 
11. Tabák AG, Herder C, Rathmann W, Brunner EJ, Kivimäki M, 2012 Prediabetes: a high-risk state for diabetes development. Lancet. 379: 2279-2290

12. Matthews DR, Hosker JP, Rudenski AS, Naylor BA, Treacher DF, Turner RC, 1985 Homeostasis model assessment: insulin resistance and beta-cell function from fasting plasma glucose and insulin concentrations in man. Diabetologia 28: 412-419.

13. National Cholesterol Education Program, National Heart, Lung, and Blood Institute, National Institutes of Health, 2002 Detection, Evaluation, and Treatment of High Blood Cholesterol in Adults (Adult Treatment Panel III) Final Report. NIH Publication No. 02-5215. Bethesda, MD: National Cholesterol Education Program, National Heart, Lung, and Blood Institute, National Institutes of Health.

14. Garrow JS, Webster J, 1985 Quetelet's index (W/H2) as a measure of fatness. Int J Obes, 9: 147-153.

15. WHO World Health Organization: Obesity: preventing and managing the global epidemic. WHO, Geneva.

16. WHO Obesity, 2000 Preventing and Managing the Global Epidemic. Report of a WHO C onsultation on Obesity. World Health Organization: Geneva.

17. Concise statistical yearbook 2009, Hellenic statistical authority, Piraeus, Greece, 2010

18. Katsilampros N, Steryotis J, Moiras N, et al, 1977 Prevalence of diabetes among glycosuric individuals in urban area of Greece. Acta Diabetologica Latina 14: 211-218.

19. Christakopoulos P, Karamanos V, Violaki M, Chaniotis D, 1976 Merikas G. The prevalence of diabetes mellitus in the rural population of Greece. Epidemiologic survey. Iatriki 29: 537-546.

20. Lionis CD, Sasarolis SM, Koutis AD, et al, 1996 Measuring the prevalence of diabetes mellitus in a Greek primary health care district. Fam Pract 13: 18-21.

21. Panagiotakos DB, Pitsavos C, Chrysohoou C, Stefanadis C, 2005 The epidemiology of Type 2 diabetes mellitus in Greek adults: the ATTICA study. Diabet Med 22: 1581-1588.

22. Melidonis AM, Tournis SM, Kompoti MK, et al, 2006 Increased prevalence of diabetes mellitus in a rural Greek population. Rural Remote Health 6: 534

23. Loizou T, Pouloukas S, Tountas C, Thanopoulou A, Karamanos V, 2006 An epidemiologic study on the prevalence of diabetes, glucose intolerance and metabolic syndrome in the adult population of the republic of Cyprus. Diabetes Care 29: 1714-1715.

24. Cowie CC, Rust KF, Byrd-Holt DD, et al, 2006 Prevalence of diabetes and impaired fasting glucose in adults in the U.S. population: National Health And Nutrition Examination Survey 1999-2002. Diabetes Care 29: 1263-1268.

25. Valdés S, Botas P, Delgado E, Diaz Cadorniga F, 2009 Mortality risk in Spanish adults with diagnosed diabetes, undiagnosed diabetes or pre-diabetes. The Asturias study
1998-2004. Rev Esp Cardiol 62: 528-534.

26. Saaristo TE, Barengo NC, Korpi-Hyovalti E, et al, 2008 High prevalence of obesity, central obesity and abnormal glucose tolerance in the middle-aged Finnish population. BMC Public Health 29: 423.

27. Beagley J, Guariguata L, Weil C, Motala AA, 2014 Global estimates of undiagnosed diabetes in adults. Diabetew Res Clin Pract 103: 150-160.

28. DECODE Study Group, 2003 Age- and sex-specific prevalences of diabetes and impaired glucose regulation in 13 European cohorts. Diabetes Care 26: 61-69.

29. Metelko Z, Pavlic-Renar I, Poljicanin T, Szirovitza L, Turek S, 2008 Prevalence of diabetes mellitus in Croatia. Diabetes Res Clin Pract 81: 263-267.

30. Van't Riet E, Alssema M, Rijkelijkhuizen JM, Kostense PJ, Nijpels G, Dekker JM, 2010 Relationship between A1C and glucose levels in the general Dutch population: the new Hoorn study. Diabetes Care 33: 61-66.

31. Rathmann W, Haastert B, Icks A, et al, 2003 High prevalence of undiagnosed diabetes mellitus in Southern Germany: target populations for efficient screening: the KORA survey 2000. Diabetologia 46: 182-189.

32. Satman I, Omer B, Tutuncu Y, et al, 2013 TURDEP-II Study Group. Twelve-year trends in the prevalence and risk factors of diabetes and prediabetes in Turkish adults. Eur J Epidemiol 28: 169-180.

33. Soriguer F, Goday A, Bosch-Comas A, et al, 2012 Prevalence of diabetes mellitus and impaired glucose regulation in Spain: the diabetes study. Diabetologia 55: 88-93.

34. Gardete-Correia L, Boavida JM, Raposo JF, et al, 2010 First diabetes prevalence study in Portugal: PREVADIAB study. Diabet Med 27: 879-881.

35. Tamayo T, Rosenbauer J, Wild SH, et al, 2014 Diabetes in Europe: An update. Diabetes Res Clin Pract 103: 206-217.

36. Cowie CC, Rust KF, Ford ES, et al, 2009 Full accounting of diabetes and pre-diabetes in the U.S. population in 1988-1994 and 2005-2006. Diabetes Care 32: 287-294.

37. Soriguer F, Goday A, Bosch-Comas A, et al, 2012 Prevalence of diabetes mellitus and impaired glucose regulation in Spain: the Di@bet.es Study Diabetologia 55: 88-93.

38. Tentolouris N, Andrianakos A, Karanikolas G, et al, 2012 Type 2 diabetes mellitus is associated with obesity, smoking and low socioeconomic status in large and representative samples of rural, urban, and suburban adult Greek populations. Hormones (Athens) 11: 458-467.

39. Koppes LLJ, Dekker JM, Hendriks HFJ, Bouter LM, Heine RJ, 2005 Moderate Alcohol Consumption Lowers the Risk of Type 2 Diabetes A meta-analysis of prospective observational studies. Diabetes Care 28: 719-725.

40. International Diabetes Federation. (IDF). Available at: http://www.idf.org/diabetesatlas

41. Kumari M, Head J, Marmot M, 2004 Prospective study 
of social and other risk factors for incidence of type 2 diabetes in the Whitehall II study. Arch Intern Med 164: $1873 \mathrm{e} 80$.

42. Jaffiol C, Thomas F, Bean K, Jego B, Danchin N, 2013 Impact of socioeconomic status on diabetes and cardiovascular risk factors: results of a large French survey. Diabetes Metab 39: 56e62.

43. Freidenberg GR, Reichart D, Olefsky JM, Henry RR, 1988 Reversibility of defective adipocyte insulin receptor kinase activity in non-insulin-dependent diabetes mellitus. Effect of weight loss. J Clin Invest 82: 1398-1406.

44. Leahy JL, 2005 Pathogenesis of type 2 diabetes mellitus. Arch Med Res 36: 197-209.

45. Colditz GA, Willett WC, Stampfer MJ, et al, 1990 Weight as a risk factor for clinical diabetes in women. Am J Epidemiol 132: 501-513.

46. Lundgren H, Bengtsson C, Blohme G, Lapidus L, Sjostrom L, 1989 Adiposity and adipose tissue distribution in relation to incidence of diabetes in women: results from a prospective population study in Gothenburg,
Sweden. Int J Obes 13: 413-423.

47. Franklin J, Thanavaro J, Ellis P, 2011 Body mass index as a guide for diagnosing prediabetes and diabetes. $\mathrm{J}$ Nurse Pract 7: 634-640.

48. Albanes D, Jones DY, Micozzi MS, Mattson ME, 1987 Associations between smoking and body weight in the US population: analysis of NHANES II. Am J Public Health 77: 439-444.

49. Voulgari C, Katsilambros N, Tentolouris N, 2011 Smoking cessation predicts amelioration of microalbuminuria in newly diagnosed type 2 diabetes mellitus: a 1-year prospective study. Metabolism 60: 1456-1464.

50. Chiolero A, Wietlisbach V, Ruffieux C, Paccaud F, Cornuz J, 2006 Clustering of risk behaviors with cigarette consumption: a population-based survey. Prev Med 42: 348-353.

51. Yusuf S, Hawken S, Ounpuu S, et al, 2004 Effect of potentially modifiable risk factors associated with myocardial infarction in 52 countries (the INTERHEART study): case-control study. Lancet 364: 937-952. 